\title{
The use of fluorocarbon radiators in the Delphi RICH detectors
}

\author{
G. Lenzen, E. Schyns, J. Thadome, J. Werner ${ }^{1}$
}

\begin{abstract}
The DELPHI experiment at LEP is equipped with Ring Imaging Cherenkov (RICH) detectors in the barrel and forward regions in order to provide particle identification over nearly the full solid angle. The use of fluorocarbons as Cherenkov radiators will be discussed.
\end{abstract}

Contribution to the First Workshop on RICH Detectors, Bari, Italy, 1993 

Introduction

Ring Imaging CHerenkov $(\mathrm{RICH})$ detectors $[1,2,3]$ are used to identify hadrons in the momentum range of $0.5 \mathrm{GeV} / c$ up to $50 \mathrm{GeV} / c$ in the DELPHI experiment $[4,5]$ at the LEP storage rings at CERN. This necessitates two radiator systems with different indices of refraction. The Cherenkov light created in liquid and gas radiators is detected by photon detectors of the time projection chamber (TPC) type. The photosensitive agent is TMAE, Tetrakis(Dimethylamino)Ethylene $\mathrm{C}_{2}\left[\left(\mathrm{CH}_{3}\right)_{2} \mathrm{~N}\right]_{4}$, with an ionization potential of $5.64 \mathrm{eV}$. Heavy fluorocarbons $\left(C_{6} F_{14}\right.$ as liquid, $C_{4} F_{10}$ and $C_{5} F_{12}$ as gas radiators $)$ serve as media in which charged particles produce Cherenkov light. These media should be transparent from $5.64 \mathrm{eV}$ to the quartz window cut-off at $7.50 \mathrm{eV}$. The above fluorocarbon media show only small chromatic aberrations. In addition these media are non-flammable, non-toxic, thermally stable and usually chemically inert.

Some properties of the DELPHI RICH radiators are given in table 1 . The index of refraction is $n$, the threshold value of $\gamma=1 / \sqrt{1-\beta^{2}}$ being defined as $\gamma_{t h}=\sqrt{1 /\left(1-1 / n^{2}\right)}$, and $\theta_{\max }$ being the Cherenkov angle for a $\beta=v / c \approx 1$ particle.

\begin{tabular}{||c|c|c|c|c|c||}
\hline & $\begin{array}{c}\text { boiling point } \\
{ }^{0} \mathrm{C}\end{array}$ & $n$ at $7 \mathrm{eV}$ & $\begin{array}{c}(\mathrm{d} n / \mathrm{dE}) * 10^{6} \mathrm{eV}^{-1} \\
6.5-7.5 \mathrm{eV}\end{array}$ & $\gamma_{t h}$ & $\begin{array}{c}\theta_{\max } \\
\mathrm{mrad}\end{array}$ \\
\hline \hline$C_{4} F_{10}$ & -2 & 1.001530 & 53 & 18.3 & 55.6 \\
$C_{5} F_{12}$ & 28 & 1.001720 & 51 & 16.9 & 59.1 \\
$C_{6} F_{14}$ & 57 & 1.283 & 9300 & 1.61 & 677.1 \\
\hline
\end{tabular}

Table 1: The properties of the fluorocarbons used in DELPHI

While $C_{6} F_{14}$ is used as liquid Cherenkov radiator medium in both Barrel and Forward RICH detectors, $C_{4} F_{10}$ serves as Forward RICH, and $C_{5} F_{12}$ as Barrel RICH gas radiator. The better UV transparency of $C_{4} F_{10}$ in comparison to $C_{5} F_{12}$ and the longer radiator length of the Forward RICH $(58 \mathrm{~cm})$ in comparison to the Barrel RICH $(<40 \mathrm{~cm})$ compensate for its lower refractive index. The number of expected photoelectrons is therefore about the same for the forward and barrel parts of the DELPHI RICH detectors. Because of its low boiling point, $C_{4} F_{10}$ requires no heating of the detector. The short radiator length of the Barrel RICH leads to the choice of the $C_{5} F_{12}$ with its higher refractive index at the price of having to maintain the detector at about $40^{\circ} \mathrm{C}$.

The DELPHI RICH fluid systems are composed of two parts:

1. The Barrel RICH fluid system [6] consisting of:

- a gas radiator volume of about $24 \mathrm{~m}^{3}$ filled with saturated perfluoropentane $C_{5} F_{12}$

- a liquid radiator circuit of 48 radiators of 51 each filled with saturated perfluorhexane $C_{6} F_{14}$, and

- a circuit of 24 pairs of drifttubes with a volume of 501 per pair containing the detector gas of $75 \%$ methane, $25 \%$ ethane, and a small amount of the photosensitive agent TMAE.

2. Two endcap Forward RICH fluid systems, each consisting of:

- a gas radiator volume of about $4 \mathrm{~m}^{3}$ of saturated perfluorobutane $C_{4} F_{10}$,

- a liquid radiator circuit of 36 radiators of about $1 \mathrm{l}$ each filled with $C_{6} F_{14}$, and

- a driftvolume circuit with 12 driftboxes of $20 \mathrm{l}$ volume each, filled with pure ethane and a small amount of TMAE. 
The three fluid systems are completely independent from each other. The liquid and gas radiator circuits of the Barrel and of both Forward RICH detectors are recirculating, while their drift gases are distributed in non-recirculating circuits.

A description of the three fluid systems can be found elsewhere [7]. The gas radiator media ( $C_{5} F_{12}$ and $C_{4} F_{10}$ ) are stored as liquids in stainless steel tanks of $500 \mathrm{l}$, and twice 1001 respectively. The appropriate liquid is evaporated into the gas radiator where it replaces slowly the radiators' nitrogen atmospheres. Turbo pumps suck the gas out of the radiators, lead it to purifiers, and then to condensers, where the gases are liquified again and fall back into the main storage tanks. The fluorocarbon gases can be purified in their liquid phases as well.

The filling of the Barrel RICH gas radiator with $C_{5} F_{12}$ is surveyed by a sonar-based device which provides an online monitor of the composition and stability of the nitrogen$C_{5} F_{12}$ gas mixture by measuring the sound velocity of the mixture [8][9]. This instrument is situated at the exit of the gas radiator. Figure 1 shows that the whole Barrel RICH gas radiator is filled with $C_{5} F_{12}$ after about 12 hours and after one volume exchange only.

The liquids $\left(C_{6} F_{14}\right)$ for both Barrel and Forward RICH detectors are also stored in stainless steel tanks, from where pumps drive the liquid through purification systems. From there each radiator tray is individually filled through up to $25 \mathrm{~m}$ long stainless steel capillaries with an internal diameter of $1.5 \mathrm{~mm}$. After having filled the radiators, the liquid $C_{6} F_{14}$ falls into $10 \mathrm{~mm}$ internal diameter nitrogen filled collector lines, and returns into the storage tank.

\section{The Transparencies of the Fluorocarbons in the UV Region}

When the DELPHI detector started data-taking in 1989, two manufacturers could deliver big quantities of perfluoropentane $C_{5} F_{12}{ }^{1)}$ and perfluorohexane $C_{6} F_{14}{ }^{2}$ ). A good UV transparency of these RICH radiators is mandatory for a high number of Cherenkov photons per event.

The quality of a batch was tested by receiving a small sample (typically 11 ) of that batch. The UV transparency was measured with the DELPHI monochromator system. These non-treated samples of liquid $C_{5} F_{12}$ and $C_{6} F_{14}$ showed a rather poor transmission in the UV region. After recirculation through a small OXISORB ${ }^{3)}$ cartridge for some hours, the samples were cleaned from oxygen and water traces, so that the UV transparency had improved considerably. The first sample of perfluorobutane $C_{4} F_{10}{ }^{4)}$ was received at the end of 1989. The very good UV gas transparency [10] after cleaning with OXISORB lead to the decision to use $C_{4} F_{10}$ as gas radiator for the Forward RICH detectors.

\footnotetext{
1) $C_{5} F_{12}: 3 \mathrm{M}$ Co. St. Paul, MN 55144, USA, under the trademark FC87 or PF5050 RTZ Chemicals, ISC Division, Bristol, UK, under the trademark PP50

2) $C_{6} F_{14}: 3 \mathrm{M}$ Co. under the trademark FC72 or PF5060

RTZ Chemicals under the trademark PP1

3) Messer Griesheim Co., D-4000 Düsseldorf, Germany

4) $C_{4} F_{10}: 3 \mathrm{M}$ Co. St. Paul, MN 55144, USA, product code DP L-12321
} 


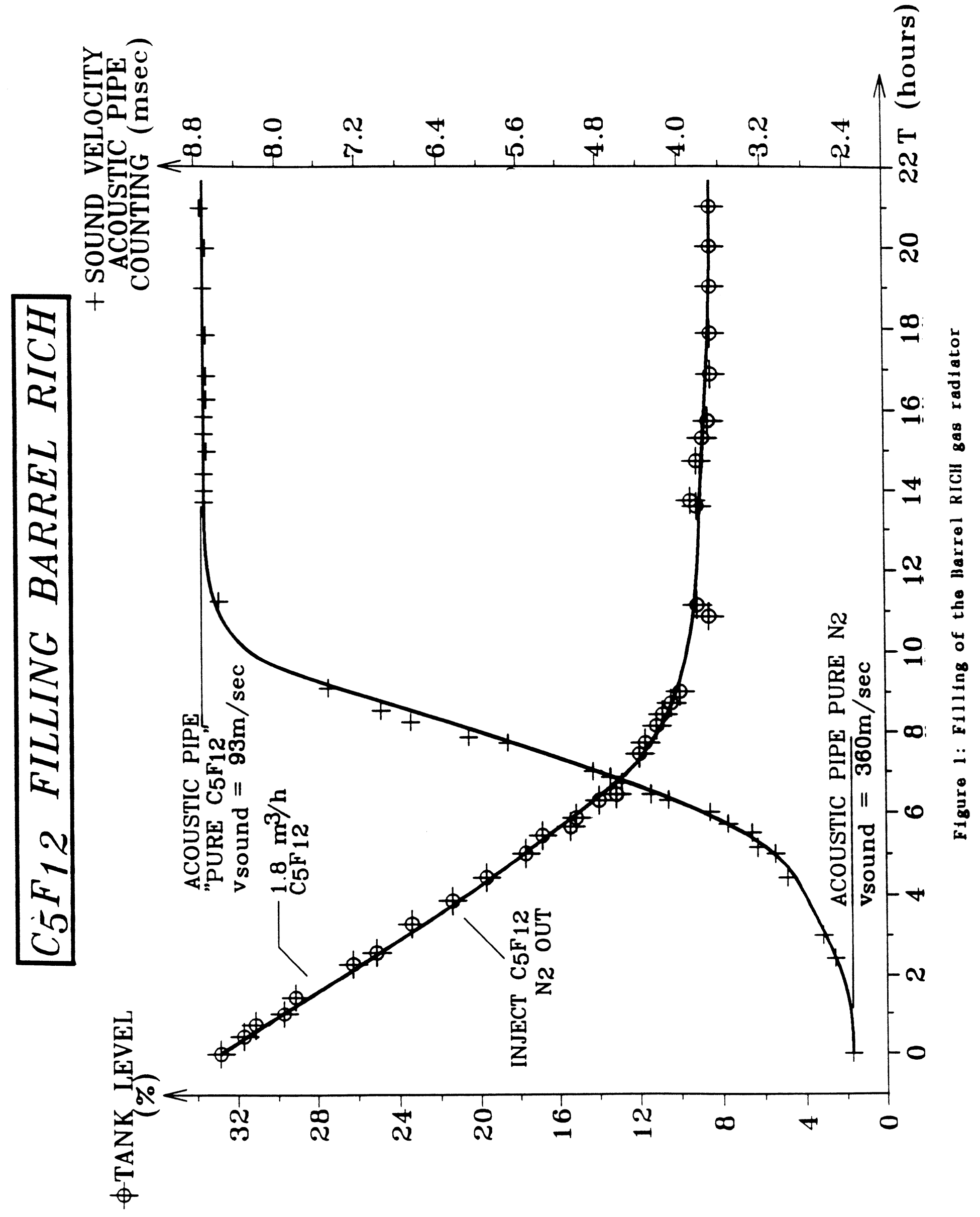




\subsection{The DELPHI RICH Monochromator System}

The quality of the Cherenkov gases and liquids has to be known on-line during data taking. Their UV transparency is measured with monochromators [9]. There are three systems in total, one for the Barrel RICH and one each for the two Forward RICH detectors.

Each monochromator system is designed to measure up to four UV transparencies simultaneously: the liquid $C_{6} F_{14}$, the gases $C_{4} F_{10}$ or $C_{5} F_{12}$, the driftgases $C_{2} H_{6}$ or a mixture $\mathrm{CH}_{4} / \mathrm{C}_{2} \mathrm{H}_{6}$, and the driftgases with TMAE.

The monochromator ${ }^{5}$ ) has $20 \mathrm{~cm}$ focal length. It is designed specifically to cover the wavelength region from about $100 \mathrm{~nm}$ to at least $250 \mathrm{~nm}$. It uses a concave holographic grating as its single reflecting surface. The optical system is similar to the Seya-Namioka design. Only a simple rotation of the grating is required to change the wavelength.

The light from a projecting deuterium lamp ${ }^{6)}$ is wavelength-selected by the grating of the monochromator. A beam divider consisting of three $\mathrm{CaF}_{2}$ windows each tilted by $45^{\circ}$ partially reflects the selected beam into three variable metal bellow cells.

The length of the cell for the $C_{6} F_{14}$ liquid can be varied by $1.5 \mathrm{~cm}$, while the short and long positions of the cells for the gas radiators and the drift gases differ by $15 \mathrm{~cm}$. The remaining part of the unreflected beam is traversing the $2.5 \mathrm{~cm}$ long variable cell which measures the TMAE loaded driftgases.

Photomultipliers ${ }^{i)}$ designed for single photon counting are installed at the end of each cell. All photomultipliers are covered with a wavelength shifter $(1 \mu \mathrm{m}$ paratherphenyl and $25 \mathrm{~nm} M g F_{2}$ ) in order to fit the spectral response to the operational region of $160 \mathrm{~nm}$ to $220 \mathrm{~nm}$.

The readout system consists of a $60 \mathrm{MHz}$ amplifier/discriminator mounted in the photomultiplier housing. It converts the anode pulse into a differential ECL signal to be read out by a LeCroy $125 \mathrm{MHz}$ counter. The monochromator systems are controlled by G64 through thinwire Ethernet communication.

Manifolds of valves connect different points of the appropriate parts of each fluid system -in general the entries and exits of the gases and liquids- with the variable monochromator cells.

\subsection{The Cleaning of Fluorocarbon Radiators and Related Problems}

Measurements with the monochromator system show that the fluorocarbon materials as delivered by the manufacturers are not sufficiently transparent in the UV region to be directly used as Cherenkov radiators. As water and oxygen are optical absorbers in the spectral range from $160 \mathrm{~nm}$ to $190 \mathrm{~nm}$, the fluorocarbons have to be cleaned by molecular sieves ${ }^{8)}$ and catalysts ${ }^{9)}$ to get rid of the water and oxygen contamination.

Nearly all fluorocarbons are sold by the manufacturers under internal trademark, rarely under their chemical composition names. Though the above fluorocarbons should be fully saturated -i.e. they should contain only carbon and fluorine- other compounds may be present which may contain additional atomic species. Only fully saturated perfluorocarbons are known to be stable and chemically inert. Only these should be cleaned by a catalyst.

\footnotetext{
5) Minuteman Model 302-VM

5) Hamamatsu $30 \mathrm{~W}$ type L 1626

7) EMI $9884 \mathrm{~B}$

8) Type $13 \mathrm{X}-8 / 2$, Chemische Fabrik Uetikon, Switzerland

9) Dispersed copper C61-1-R, Catalyst and Chemicals, Brussels, Belgium
} 
In 1992 it was found that some batches of perfluorohexane $\left(C_{6} F_{14}\right)$ would chemically react with the catalyst which resulted in a large unacceptable loss of UV transparency. Chemical and physico-chemical techniques, i.e. distillation, UV-visible and FT-IR spectrometry were used to identify the causes of this chemical reaction mechanism. It could be shown that the reaction is due to a contamination with incompletely fluorinated molecules (general formulae $C_{n} H_{x} F_{2 n-x}$ ) [11]. As the violent reaction between the catalyst and the oxygen in the gas or liquid could endanger the optimal operation of the detector, a nonaggressive inert cleaning system to remove both oxygen and water from perfluorohexane was successfully tested, the capillary membranes [12]. This device consists of a bundle of capillaries mounted in a stainless steel container. The liquid to be cleaned passes through the capillaries and the permeating substances (oxygen and water) are removed by pumping on the surrounding space of the capillaries. The working principle is based on permeating properties of different molecules with various kinetic diameters.

Two other non-aggressive cleaning methods for removing oxygen from liquid $C_{5} F_{12}$ and $C_{6} F_{14}$ were tested and shown to work: flushing the liquid with nitrogen and ultrasonic degassing [13].

Recently delivered batches of $C_{5} F_{12}$ and $C_{6} F_{14}$ show already a very good UV transparency. Therefore only molecular sieve cartridges of about $5 \mathrm{~kg}$ are used to improve the quality of the liquids.

\subsection{Results}

All three fluorocarbons $\left(C_{4} F_{10}, C_{5} F_{12}\right.$, and $\left.C_{6} F_{14}\right)$ were measured with the same monochromator system in the spectral region from $160 \mathrm{~nm}$ to $220 \mathrm{~nm}$. The UV transparencies of $C_{4} F_{10}$ and $C_{5} F_{12}$ were measured with the $15 \mathrm{~cm}$ variable gas cell at $40^{\circ} \mathrm{C}$ and atmospheric pressure, while the UV transparencies of $C_{6} F_{14}$ and liquid $C_{5} F_{12}$ were determined with the $1 \mathrm{~cm}$ liquid cell of the monochromator system at $25^{\circ} \mathrm{C}$ and atmospheric pressure.

The integrated measured transparency curve may indicate a good quality of the fluorocarbons. A $100 \%$ transparent liquid or gas in the spectral range from $1600 \AA$ to $2200 \AA$ would lead to an integral value of $600 \AA$. Integral values of more than $400 \AA$ for the liquid transparencies and of more than $520 \AA$ for the gas transparencies are regarded as indications for good Cherenkov radiator qualities.

Typically, after a week of circulation of the gases and liquids through the radiators and purification systems there are no dramatic differences anymore between the qualities at the entrances and exits of the RICH detectors.

Figure 2 gives UV transparencies for the three fluorocarbons used in the DELPHI RICH detector after recirculation of one week. The bottom curve in each plot of figure 2 shows the product of the measured transparency curve with the absolute TMAE quantum efficiency curve [14]. The numbers of the integrated transparency curve and the integrated product of the transparency curve with the absolute TMAE quantum efficiency curve is given inside each subplot of figure 2 . 

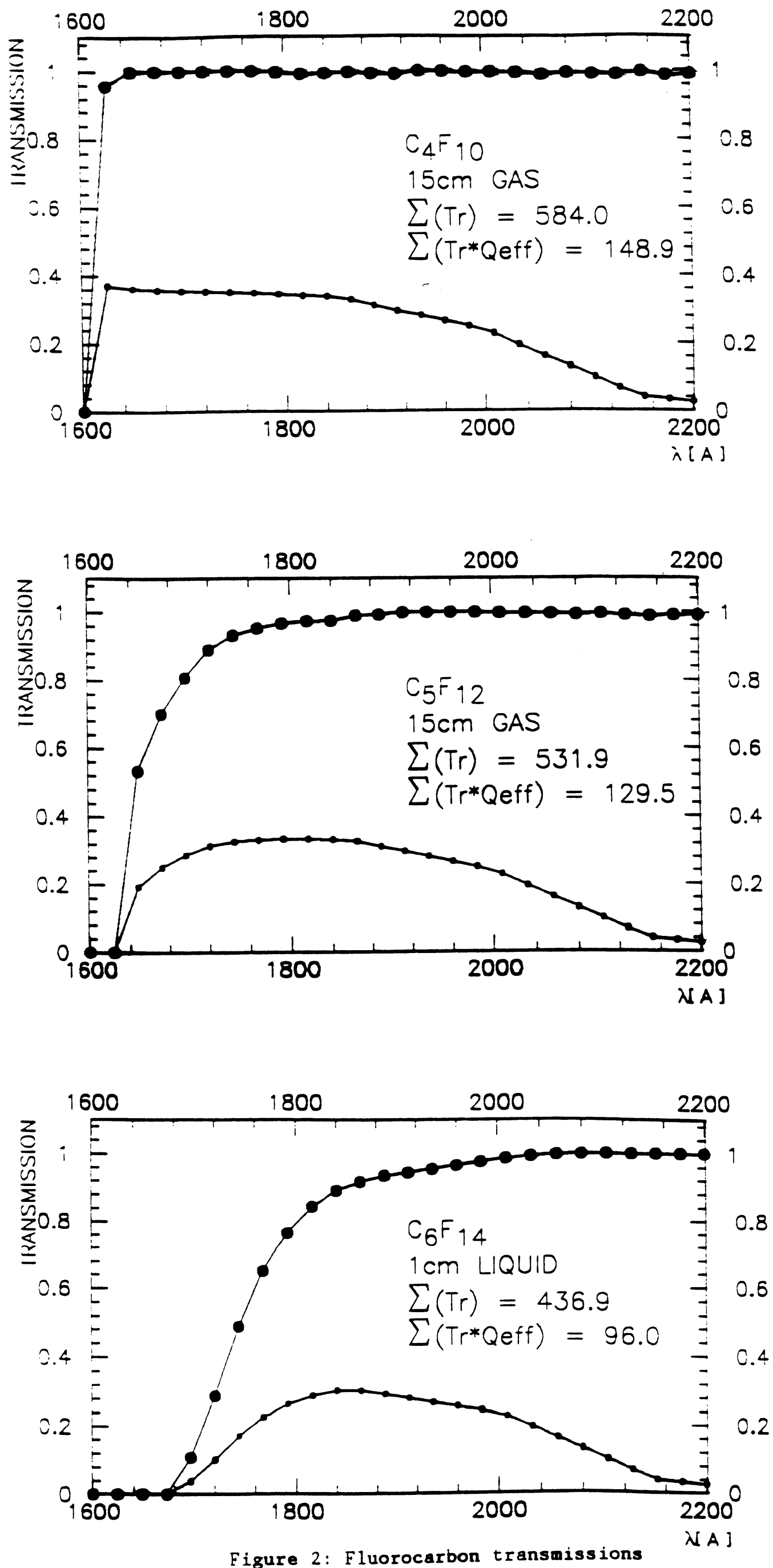


\subsection{Figure of Merit $\left(N_{0}\right)$}

The above UV transparency measurements can be used to estimate the expected number of photoelectrons $N_{p e}$ in a RICH detector [15], which is determined by

$$
N_{p e}=N_{0} L \sin ^{2} \theta
$$

where $N_{0}$ is the so-called figure of merit which is defined by

$$
N_{0}=370 \int \eta(E) Q(E) R(E) T(E) d E\left[\mathrm{~cm}^{-1}\right]
$$

where:

$L$ is the radiator length in $\mathrm{cm}$,

$\theta$ is the Cherenkov angle, depending on the refractive index $n$ (taken at $7 \mathrm{eV}$ ),

$\eta(\mathrm{E})$ is the electron detection efficiency,

and $\mathrm{Q}(\mathrm{E}), \mathrm{R}(\mathrm{E})$ and $\mathrm{T}(\mathrm{E})$ are the absolute quantum efficiency for photon conversion, the reflectivity of the mirror and the transmission of several detector components (radiator gas, quartz windows, drift gas, etc.) respectively.

Here $N_{0}$ is defined under the assumption of perpendicular particle incidence, unlimited acceptance and $\eta(\mathrm{E})=1$.

All wavelength dependent transmissions of materials were measured for perpendicularly incident UV-light, whereas in the actual experiment the angle of incidence varies widely. In addition dead space, internal total reflection in the quartz windows, conversion depth and attenuation length of TMAE, dead time of the preamplifiers and efficiency of the cathodes of the MWPC, Lorentz angle, and last but not least the reconstruction efficiency of Cherenkov photoelectrons in hadronic jets have to be taken into account. All these factors may lead to a significant loss in the number of photoelectrons per event. Only a detailed Monte Carlo program can reliably predict this number.

Table 2 presents the results for the figures of merit for the DELPHI Barrel and Forward RICH detectors, when only the measured qualities of the detector elements, like transmissions of the radiator gas and liquid, transmission of quartz, transmission of detector drift gas, mirror reflectivity, and TMAE quantum efficiency are considered. The electron detection efficiency $\eta(\mathrm{E})$ was set to 1 , and the velocity of the charged particle is $\beta \approx 1$.

\begin{tabular}{||c|c|c||}
\hline$N_{0}\left[\mathrm{~cm}^{-1}\right] / N_{p e}$ & $\begin{array}{c}\text { Barrel RICH } \\
C_{5} F_{12} \text { and } C_{6} F_{14}\end{array}$ & $\begin{array}{c}\text { Forward RICH } \\
C_{4} F_{10} \text { and } C_{6} F_{14}\end{array}$ \\
\hline gas radiator & $81.6 / 11.1$ & $89.6 / 15.6$ \\
liquid radiator & $70.0 / 27.5$ & $70.0 / 27.5$ \\
\hline
\end{tabular}

Table 2: The figures of merit $N_{0}$ and the number of expected photoelectrons $N_{p e}$ under the assumption of full electron detection efficiency

The mean number of detected photoelectrons per muon event with momentum $>40 \mathrm{GeV} / c$ in the DELPHI experiment is 8 for the Barrel $\mathrm{RICH}$ gas radiator, and 13 for the Barrel RICH liquid radiator. In the Forward RICH there are 12 photoelectrons detected from the gas radiator, and 8 photoelectrons from the liquid radiator. These numbers are closely predicted by detailed Monte Carlo studies [3], which take into account all the above mentioned effects. The number of detected photoelectrons follows Poisson statistics. The experimental resolutions are close to those given by the Monte Carlo calculations. 


\section{Acknowledgements}

We would like to thank all our collaborators in DELPHI for their encouragement. We are especially grateful for the constant interest and support by U.Amaldi and J.Drees. M.Bosteels' contribution to the layout of the RICH fluid system is acknowledged with thanks. We express our thanks to S.Ilie for his guidance in the fluorocarbon purification. G.Hallewell is thanked for many fruitful discussions. 


\section{References}

[1] J.Séguinot, T.Ypsilantis: Nucl.Instr. and Meth. 142 (1977) 377

[2] E.G.Anassontzis et al.: Nucl.Instr. and Meth. A323 (1992) 351

[3] W.Adam et al.: Nucl.Instr. and Meth. in preparation

[4] DELPHI Technical Proposal, CERN/LEPC 83-3 (1983)

[5] P.Aarnio et al.: Nucl.Instr. and Meth. A303 (1991) 233

[6] M.Bosteels, G.Lenzen: Internal Report, DELPHI 86-77 RICH-20 (1986)

[7] W.Adam et al.: Contribution to this workshop

[8] G.Hallewell et al.: SLAC-PUB-4122, January 1987

[9] J.Werner: Diploma thesis WUB-89-3, January 1989

[10] G.Lenzen, J.Werner: Internal Report, October 1990

[11] S.Ilie, G.Lenzen: Internal Report, DELPHI 93-33 RICH-54 (1993)

[12] M.Bosteels, S.Ilie: Technical Note MT-SM/93-02, CERN, March 1993

[13] M.Danielsson, H.-J. Gerber: to be submitted to Nucl.Instr. and Meth.

[14] R.A.Holroyd et al.: Nucl.Instr. and Meth. A261 (1987) 440

[15] G.Lenzen, J.Werner: Internal Report, DELPHI 87-94 RICH-29, December 1987 